\title{
Análisis de falla en soldadura disímil (Duplex 2202-Austenítico 201LN).
}

\author{
Failure analysis in dissimilar weld (Duplex 2202-Austenítico 201LN). \\ César Mendoza-Gómora ${ }^{a}$, Carlos Ernesto Borja-Soto ${ }^{b}$
}

\begin{abstract}
:
Mechanical behavior of welded metallic components is influenced by internal defects in the weld, such as inclusions and porosity. This work analyzes by means of visual inspection and optical microscopy a pair of dissimilar joints realized by the GMAW process. The study permitted to associate the lack of fusion to the presence of porosity.
\end{abstract}

Keywords:

Porosity, GMAW, dissimilar weld, cracks.

Resumen:

El comportamiento mecánico de los componentes soldados está influenciado por defectos internos en la soldadura, tales como inclusiones y porosidad. Este trabajo presenta y analiza mediante inspección visual y microscopía óptica dos uniones disímiles realizadas por el proceso de soldadura GMAW. El estudio permitió asociar la falta de fusión a la presencia de la porosidad.

Palabras Clave:

Porosidad, GMAW, soldadura disímil, grietas.

\section{Introducción}

Las impurezas en la superficie de los materiales, tales como óxido, polvo y grasas presentes afectan considerablemente el comportamiento mecánico de las soldaduras. Al quedar atrapados estos contaminantes durante el proceso de fusión y solidificación causan porosidad.

La porosidad es un defecto presente en los procesos de soldadura debido a la formación de gases que podrían quedar atrapados al solidificar, al igual que por no tener un control en la limpieza de los materiales a emplear, ya que contienen impurezas que pueden quedar atrapadas. Como se mencionó, la porosidad se puede presentar en diversos tipos de soldadura, X. Zhan y colaboradores estudiaron la porosidad generada durante el proceso de soldadura híbrido láser-MIG y concluyeron que la porosidad es causada por los gases disueltos, así como por la evaporación del elemento $\mathrm{Mg}$ y se encuentra mayormente en la parte superior del cordón de soldadura.
Adicionalmente indicaron que la porosidad generó una disminución de la resistencia a la tensión. 1

Por otro lado, Madison y Aagesen caracterizaron cuantitativamente la porosidad en uniones de acero inoxidable 304 realizadas por el proceso de soldadura por láser. Los investigadores variaron el calor de aporte por medio de un incremento en la velocidad de avance, esto generó una rapidez de enfriamiento distinta en cada una de las soldaduras. Con estos cambios determinaron que la frecuencia, forma y volumen de los poros varían directamente con los cambios en los parámetros de soldadura y que a velocidades de desplazamiento elevadas las impurezas y el gas de protección pueden influir notoriamente en la porosidad. 2

La humedad presente en los materiales a unir influye en el grado de porosidad en el cordón de soldadura. Al superarse la temperatura de ebullición del agua en el proceso de soldadura, se genera vapor que puede quedar atrapado. Si la temperatura es elevada los átomos de

Autor de Correspondencia, Universidad Autónoma del Estado de Hidalgo, Escuela Superior Ciudad Sahagún, Email: cesar_mendoza@uaeh.edu.mx

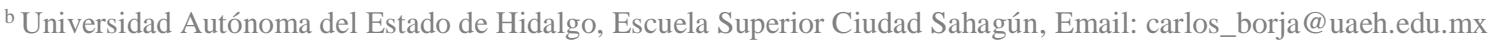


hidrógeno se separan del oxígeno dando paso al fenómeno de fragilización por hidrógeno. 3, 4, 5

El presente trabajo analiza un caso de estudio de dos soldaduras disímiles proporcionadas por una empresa, la cual indicó que durante la operación de este tipo de uniones se genera la falla de los componentes soldados. Al ser muestras proporcionadas se desconoce su historial, es decir, no se cuenta con los datos de las variables operativas utilizadas, así como los cuidados previos antes de realizar la soldadura.

\section{Material y métodos}

Se conoce que las soldaduras de filete proporcionadas por la empresa fueron realizadas por el proceso de soldadura GMAW y fueron empleadas placas de acero inoxidable austenítico 201 LN y acero inoxidable dúplex 2202. Como material de aporte se utilizó un electrodo de acero inoxidable dúplex 2209. La Tabla 1 muestra la composición química de los materiales utilizados.

Tabla 1. Composición química de los materiales empleados (\% en peso).

\begin{tabular}{lcccccccc}
\hline & $\mathbf{C r}$ & $\mathbf{N i}$ & Mo & Mn & Si & Cu & N & C \\
\hline 201 LN & 16.02 & 4.41 & - & 6.72 & 0.53 & 0.309 & 0.208 & 0.028 \\
UR 2202 & 22.87 & 1.22 & 0.26 & 1.14 & 0.47 & - & 0.237 & 0.028 \\
ER-2209 & 23.01 & 8.6 & 3.1 & 1.6 & 0.8 & $<0.1$ & 0.15 & 0.01 \\
\hline
\end{tabular}

${ }^{*}$ Composición química nominal.

Las muestras fueron preparadas metalográficamente. Primeramente se desbastaron con lijas de diferente granulometría $(250,400,600,800,1000,1200,1500$ y 2000). Posteriormente fueron pulidas acabado espejo con pasta de diamante de 3 y $1 \mu \mathrm{m}$. La Figura 1 muestra el equipo utilizado para este último paso.

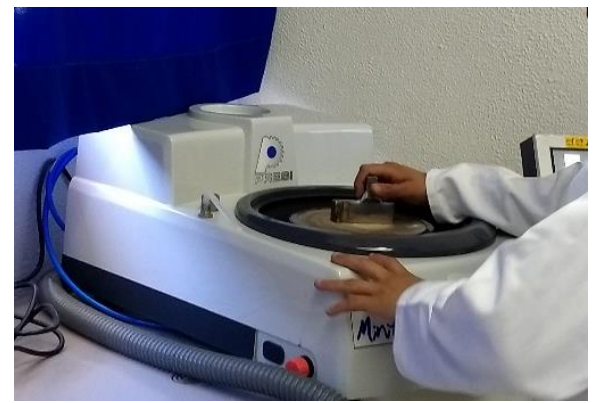

Figura 1. Proceso de pulido acabado espejo.

Con el propósito de revelar las micro y macroestructura del cordón de soldadura, las muestras fueron sumergidas en el reactivo vilella por un tiempo de $5 \mathrm{~min}$.

\section{Análisis y discusión de resultados}

La Figura 2 muestra la macroestructura de cada una de las probetas. En la muestra $A$ el acero dúplex tiene un espesor de $7.94 \mathrm{~mm}$ mientras que para la muestra $B$ es de $6.4 \mathrm{~mm}$. En ambas muestras el acero austenítico cuenta con un espesor de $20.64 \mathrm{~mm}$.
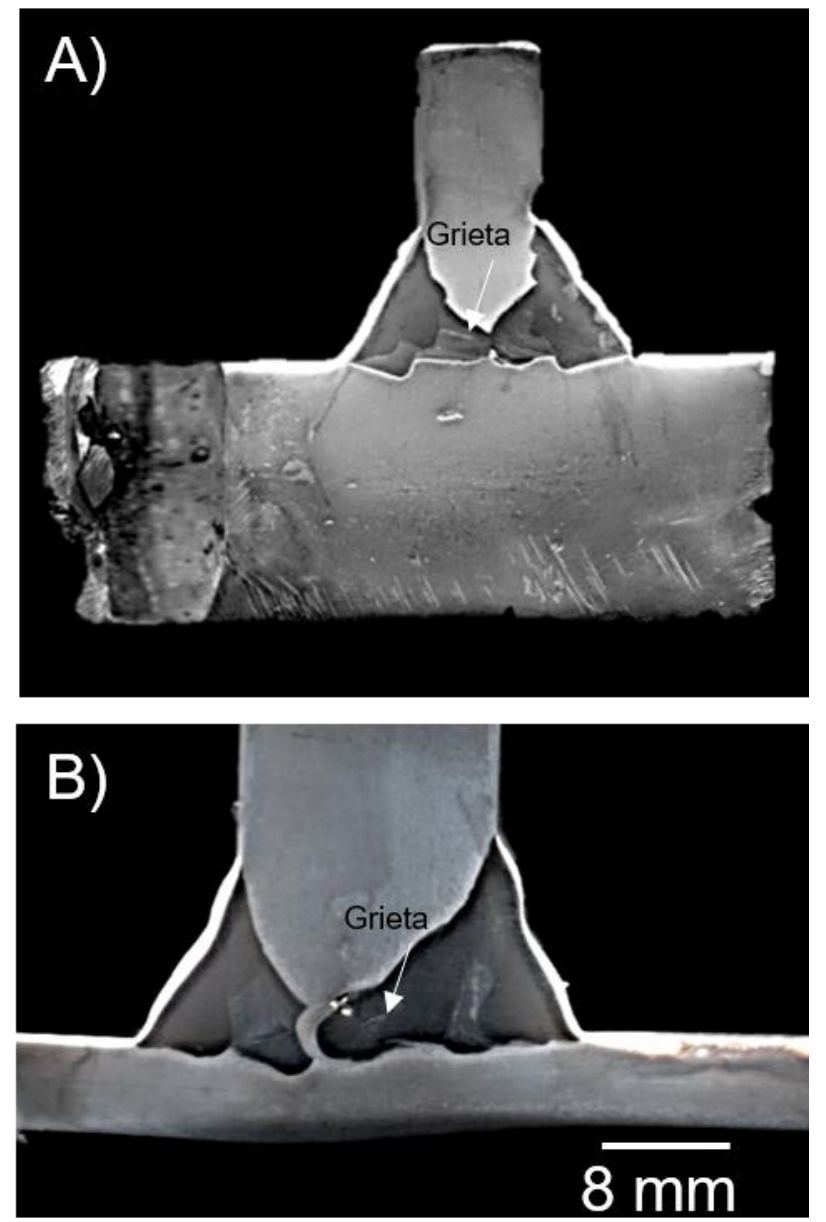

Figura 2. Macroestructura de las uniones proporcionadas.

En la probeta A se puede observar el número de pasos de soldadura, 4 para el lado derecho y 4 para el lado izquierdo. Puede verse que entre los cordones de raíz existe un pequeño poro, sin embargo, el caso más detrimental a nivel macroscópico es la grieta que se presenta del lado izquierdo. La Figura 3 muestra la grieta de la muestra A. Como se puede observar la grieta inicia al centro y se propaga hacia los extremos. Durante el proceso de soldadura de aceros inoxidables se presenta la precipitación de las fases intermetálicas $\sigma$ y $x[6,7]$ que son frágiles. Por otro lado, durante la fusión y solidificación se producen esfuerzos residuales que en conjunto con las fases precipitadas y la porosidad propician la fractura.

Con respecto a la probeta $B$ puede verse que la soldadura también fue realizada por pasos; 4 cordones de cada lado y entre los pasos de raíz se presenta nuevamente falta de fusión (poro). Esta soldadura muestra una grieta del lado 
derecho, al igual que la probeta $A$ y que está atribuida a los fenómenos antes mencionados.

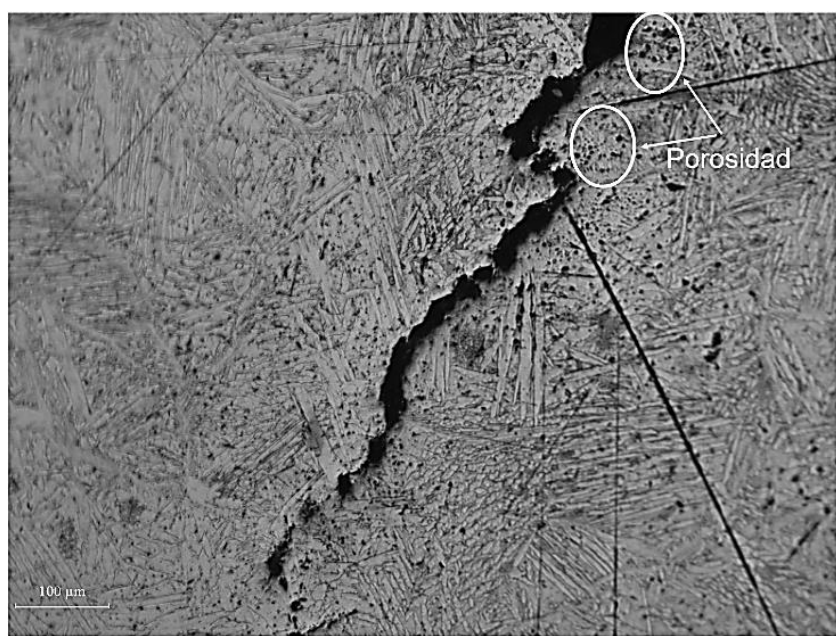

Figura 3. Grieta en de la muestra $A$.

La Figura 4 muestra la interfase entre el cordón de soldadura y el material base inferior del lado izquierdo de la Figura 2 A) y la Figura 5 muestra la interfase entre el cordón de soldadura y el material base superior de la misma muestra. Como se puede observar, el mayor daño en la junta soldada ha sido en la interfase del cordón de soldadura y los materiales base empleados. Es evidente la existencia de una gran cantidad de poros (zonas oscuras) sobre la interfase. La porosidad no se encuentra en la parte superior del cordón de soldadura por lo que se descarta la idea de que se deba a la evaporación de elementos de aleación o humedad. Durante un proceso de soldadura se requiere que haya una limpieza del metal base antes de comenzar el cordón, los contaminantes presentes al quemarse con el calor de aporte generan humos generando porosidad. Por la zona en que se encuentra y debido a que la porosidad se distribuye por toda la interfase, se concluye que no se realizó la limpieza adecuada antes de aplicar los cordones de soldadura, lo que generó la falta de fusión y en consecuencia una soldadura no aceptable.

Con respecto a la muestra $\mathrm{B}$, la Figura 6 muestra la interfase superior entre los cordones de soldadura y el metal base. Puede verse claramente que es una interfase sana, es decir, no presenta porosidad como la muestra $A$.

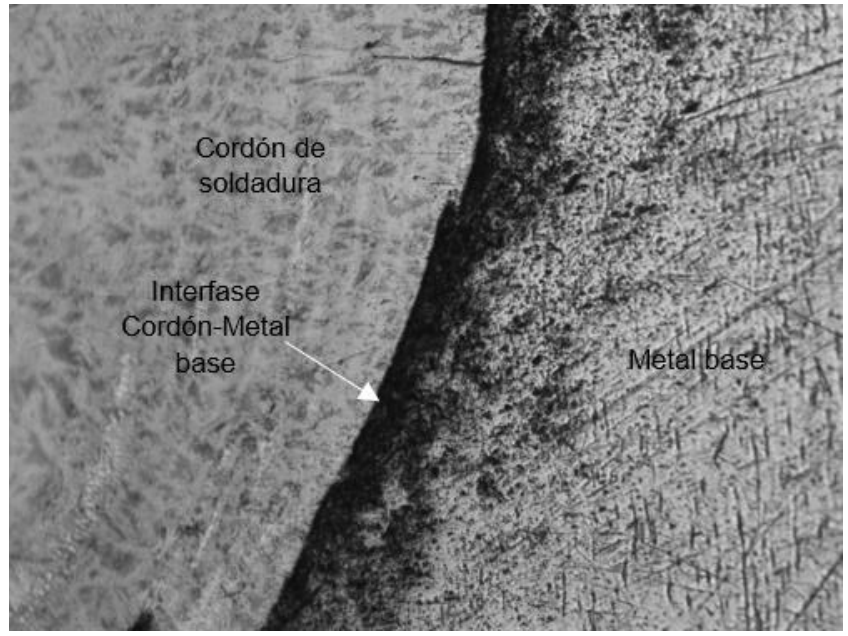

Figura 4. Interfase inferior cordón de soldadura-metal base de la muestra $A$.

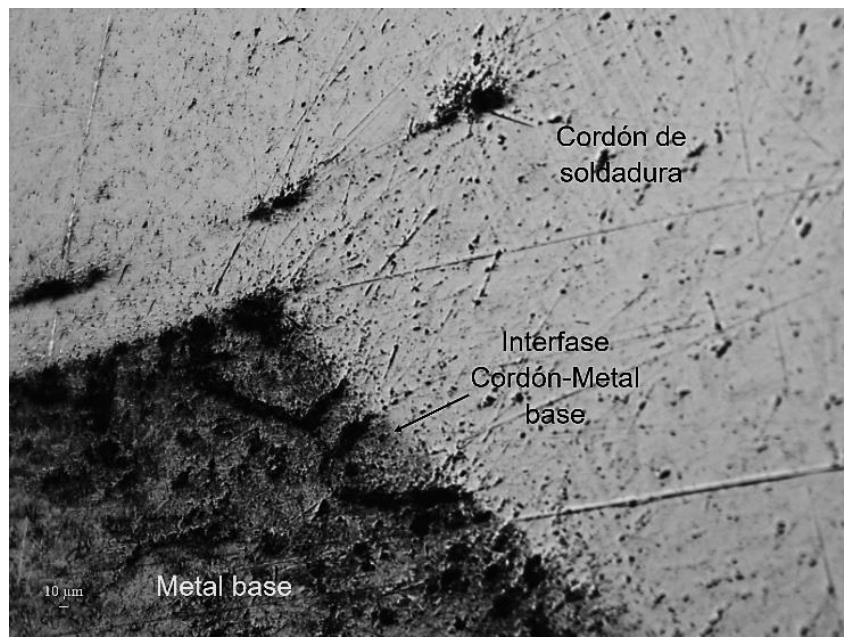

Figura 5. Interfase superior cordón de soldadura-metal base de la muestra $A$.

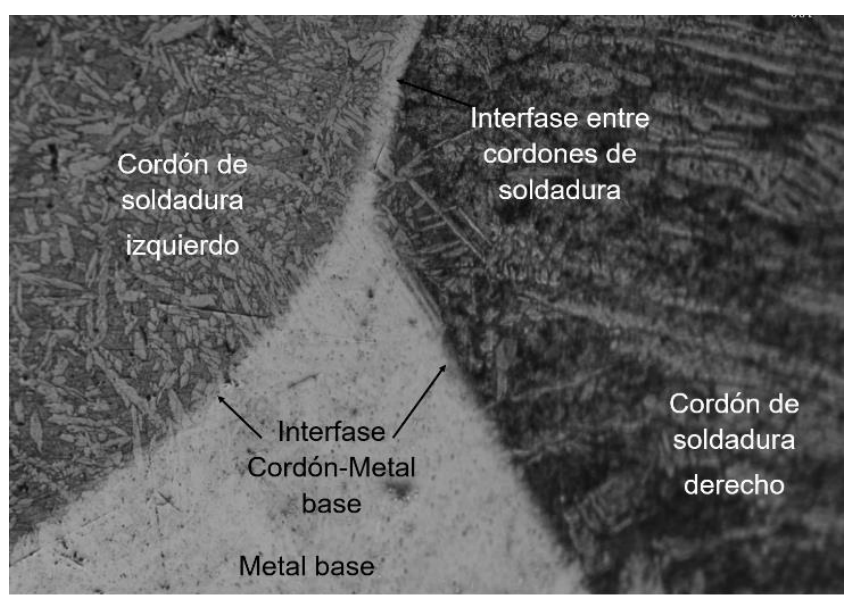

Figura 6. Interfase superior cordón de soldadura-metal base de la muestra $B$. 
Por otro lado, la Figura 7 muestra la interfase inferior cordón de soldadura-metal base. Como se observa, esta interfase muestra porosidad como la muestra $A$, sin embargo es en menor cantidad. Por lo que estudios como cuantificación de tamaño y distribución de poros tendría que realizarse para poder atribuir si es una junta sana 0 no. A nivel laboratorio este tipo de problemas no se presenta, sin embargo, a nivel industrial por la alta demanda el operario no pone énfasis en la limpieza de los materiales por lo que a menor o mayor escala las imperfecciones están presentes.

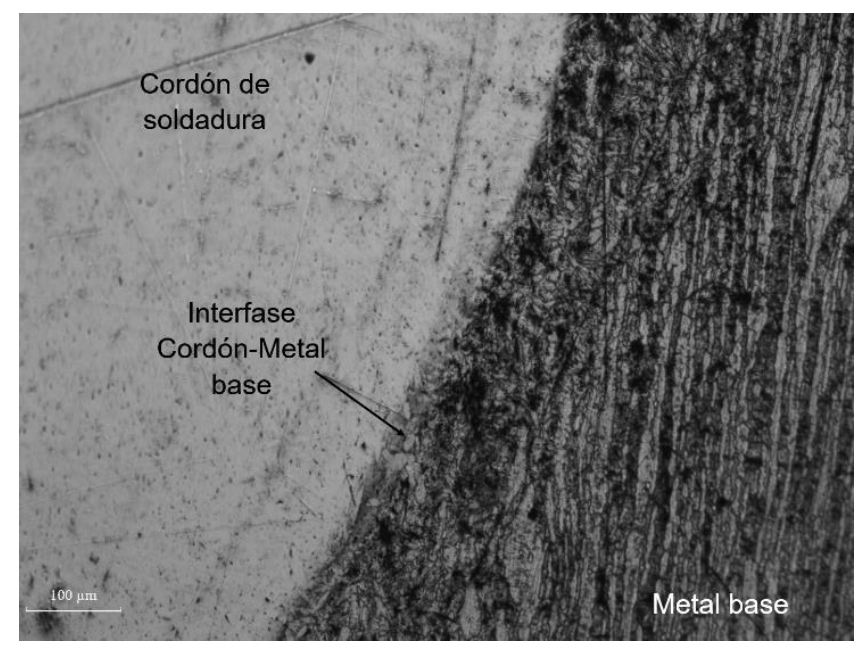

Figura 7. Interfase inferior cordón de soldadura-metal base de la muestra $B$.

\section{Conclusiones}

Las muestras analizadas fueron realizadas por soldaduras de pasos. El mayor daño que presentan las muestras es falta de fusión en las interfaces cordón de soldadurametal base a causa de porosidad y, se atribuye a falta de limpieza durante la aplicación de los cordones de soldadura. La muestra B presenta menor cantidad de porosidad que la muestra $A$ por lo que es necesario realizar cuantificación de distribución y tamaño de poro para identificar si es una soldadura sana.

\section{Referencias}

[1] Zhan X, Zhao Y, Liu Z, Gao O, Bu H. Microstructure and porosity characteristics of 5A06 aluminum alloy joints using laser-MIG hybrid welding. Journal of Manufacturing Processes, 2018; 35: 437-445.

[2] D. Madison JD, Aagesen LK. Quantitative characterization of porosity in laser welds of stainless steel. Scripta Materialia, 2012; 67: 783-786.

[3] Gou G, Zhang M, Chen H, Chena, Li JP, Yang YP. Effect of humidity on porosity, microstructure, and fatigue strength of A7N01S-T5 aluminum alloy welded joints in high-speed trains. Materials and Design, 2015; 85: 309-317.
[4] Guoa N, Fu Y, Xing X, Liu Y, Zhao S, Feng J. Underwater local dry cavity laser welding of 304 stainless steel. Journal of Materials Processing Tech.. 2018; 260: 146-155.

[5] Dwivedy SK., Vishwakarma M. Hydrogen embrittlement in different materials: A review. International Journal of Hydrogen Energy. 2017; $1-14$.

[6] Pohl M, Storz O, Glogowski T. Effect of intermetallic precipitations on the properties of dúplex stainless Steel. Materials Characterization. 2007; 58: 65-71.

[7] Escriba DM, Materna-Morris E, Plaut RL, Padilha AF. Chi-phase precipitation in a dúplex stainless Steel. Materials Characterization, 2009; 60: 1214-1219. 